\title{
Ende der repräsentativen Demokratie? \\ Eine Staatsform vor der Alternative ihrerselbst
}

\author{
Otto Depenheuer
}

\section{A. Demokratiedämmerung?}

Die Demokratie des Grundgesetzes versteht sich als „wehrhafte“. ${ }^{1}$ Nie wieder sollen die Feinde der Demokratie diese freiheitlichste je auf deutschem Boden realisierte Staatsform mißbrauchen dürfen. Zahlreich die Vorkehrungen, das Trauma der Deutschen von 1933 - die „legale Machtergreifung" - vorbeugend zu verhindern. Doch wie so oft in der Geschichte: die Gefahr kommt nicht von dem Feind, den man vermutet und gegen den man sich gewappnet hat. Gefahr erwächst der grundgesetzlichen Demokratie still, allmählich, fast unmerklich, strukturell unpolitisch, als Konsequenz geschichtlicher Entwicklung: unaufhörliche Komplexitätssteigerungen der Entscheidungslagen, entsprechend zunehmende Ausbildung technokratischer Subsysteme, potenziert durch überstaatliche Problembewältigungsherausforderungen und internationale Normgenerierung. Parlamentarisch zu bewältigende Entscheidungslagen werden parallel dazu immer komplexer und diffuser. Regelmäßig verbunden mit extremem zeitlichem Entscheidungsdruck führen diese Rahmenbedingungen im Ergebnis zu einer weitgehenden faktischen Entmachtung der nationalen Parlamente. ${ }^{2}$ Aber nicht nur das Substrat des demokratischen Entscheidungs-

1 Vgl. Markus Thiel (Hg.), Wehrhafte Demokratie, 2003; Jürgen Becker, Die wehrhafte Demokratie des Grundgesetzes, in: Josef Isensee/Paul Kirchhof (Hg.), Handbuch des Staatsrechts (HStR), Bd. VII (1992), § 167; Otto Depenheuer, Wehrhafte Republik, in: Rolf Gröschner/Oliver W. Lembcke (Hg.), Freistaatlichkeit. Prinzipien eines europäischen Republikanismus, 2011, S. $211 \mathrm{ff}$.

2 Ausführlich Analyse: Quirin Weber, Parlament - Ort der politischen Entscheidung?, Basel 2011. Vgl. auch Otto Depenheuer, Demokratie zwischen Effizienz und Legitimation, in: Günther Nonnenmacher/Andreas Rödder (Hg.), Kapitalismus und Demokratie, Vierte Tendenzwende-Konferenz der FAZ am 15. und 16. November 2012 in Berlin, 2013, S. 24-30; ders. Demokratie und Staatsvolk. Die Bindung des Wahlrechts an die Staatsangehörigkeit, in: Gornig/Horn/Murswiek (Hg.), Nationales Wahlecht und internationale Freizügigkeit, 2015, S. 39 ff. 
prozesses verschwimmt im Dunstkreis maximaler Komplexität, zeitgleich wird auch das Subjekt der Demokratie immer undeutlicher. Der Begriff des Volkes ist längst nicht mehr so klar wie er einmal erschien: im Zeitalter von Internationalisierung und Globalisierung, von supranationaler Integration und immer größeren weltweiten Migrationsströmen verliert der demokratisch verfaßte Nationalstaat den Quellgrund, auf dem eine demokratische Organisation politischer Macht ihrer Begrifflichkeit nach beruht: den Demos, d.h. das Volk. ${ }^{3}$ Dieser Befund bezeichnet ein demokratietheoretisches Problem. Wenn Demokratie Herrschaft des Volkes ist, dann kann es nicht folgenlos bleiben, wenn das Volk als prinzipiell feste Größe fragwürdig wird, seine Substanz sich ändert und eine zahlenmäßig erhebliche Diskrepanz zwischen dem deutschen Volk als der Summe aller Staatsangehörigen einerseits und der innerhalb des Bundesgebietes ansässigen Bevölkerung andererseits festzustellen ist. Was wird aus der „Herrschaft des Volkes“, wenn der Begriff des Volkes ,undeutlich“ wird, der spezifische Legitimationsquell der Demokratie verdunstet, diffus wird, nicht mehr abgrenzbar ist? Der Bundestag hat den angezeigten Wandel baulich bereits verarbeitet: er sieht sich nicht mehr nur - wie an der historischen Stirnfront des Reichstagsgebäudes bekundet - „dem Deutsche Volke“ verpflichtet, sondern - etwas versteckt im Innenhof - ebenso auch „der Bevölkerung". ${ }^{4}$ Der Beitrag von Wessel le Roux zielt in gleicher Weise darauf $\mathrm{ab}$, die gegenwärtigen Probleme der repräsentativen Demokratie in Südafrika durch eine Ersetzung des Volksbegriffs als Kern der Demokratie durch den Begriff der Bevölkerung, also die Ersetzung der Staatsangehörigkeit durch die der Betroffenheit der im Staatsgebiet Lebenden, zu ersetzen. Gegenüber derartigen vorschnellen Antworten auf einen Wandel der realen Verhätnisse scheint mir ein gehöriges Maß an Skepsis angebracht. Um diese zu verdeutlichen, darf ich zunächst die Problemanalyse noch etwas verbreitern und vertiefen. Insoweit möchte ich daran erinnern, daß nur ein in der Wirklichkeit vorfindbares, bestimmtes oder bestimmbares Kollektiv repräsentiert werden kann. Sodann soll die unverzichtbare politische Dimension des Problems der Unterscheidung zwischen Staaten und ihren

3 Josef Isensee, Abschied der Demokratie vom Demos. Ausländerwahlrecht als Identitätsfrage für Volk, Demokratie und Verfassung, in: Dieter Schwab u.a. (Hg.), Staat, Kirche, Wissenschaft in einer pluralistischen Gesellschaft. Festschrift für Paul Mikat, 1989, S. 705.

4 Dokumentation der öffentlichen Debatte bei Michael Diers/Kaspar König (Hg.), Der Bevölkerung, 2000. 
Staatsvölkern zumindest ansatzweise rekonstruierend skizziert werden. Am Ende soll schließlich ein durchaus unprätentiöser Lösungsvorschlag vorgestellt werden, der im Ergebnis wenig aufregend darauf hinzielt, es im Wesentlichen so zu belassen, wie es gegenwärtig ist.

\section{B. Wen repräsentiert die repräsentative Demokratie?}

Im Kontext von Globalisierung und Migrationsbewegungen, wie wir sie gegenwärtig erleben, stellt sich in der Tat die Frage, welchen rechtlichen Status die Zuwanderer in den Zuwanderungsstaaten haben sollen. Da sie keine Staatsangehörigen und deshalb von Wahlen ausgeschlossen sind, ${ }^{5}$ werden sie demokratisch nicht repräsentiert. Das rechtspolitische Votum vieler Beobachter geht deshalb dahin, daß sie, weil sie von den Entscheidungen des Aufenthaltsstaates betroffen seien, repräsentiert werden sollten, deshalb Bürgerstatus erlangen sollten, damit sie derart auch zum demokratischen Souverän gehören, folglich integriert und repräsentiert sind. Diese modulare Problemlösungsstrategie erscheint indes etwas unterkomplex: sie trägt den Voraussetzungen jeder Repräsentation nicht hinreichend Rechnung, nämlich dem Bestehen einer Entität, die überhaupt repräsentiert werden kann.

\section{Das Volk als „unsichtbares Sein“}

Carl Schmitt hat in seiner Verfassungslehre ${ }^{6}$ den Begriff der Repräsentation wie folgt definiert: „Repräsentieren heißt, ein unsichtbares Sein durch ein öffentlich anwesendes Sein sichtbar zu machen und zu vergegenwärtigen." Repräsentation bedeutet also, ein unsichtbares Sein, nämlich das Sein des Staates oder des Volkes durch ein öffentlich anwesendes Sein sichtbar zu machen, zu vergegenwärtigen. Ähnlich formulierte Heinrich Triepel, „Repräsentieren heißt die Darstellung eines schwer erkennbaren Seins durch eine leichter erfaßbare Wirklichkeit."7 Diese beiden exemplarischen Definitionen verweisen also auf etwas, das überhaupt repräsentiert werden kann: das Dasein einer abstrakten Entität. Herkömmlich erfolgt in-

5 BVerfGE 83, 37, 50 ff. - Ausländerwahlrecht.

6 Carl Schmitt, Verfassungslehre, 1928, S. 209.

7 Heinrich Triepel, Delegation und Mandat im öffentlichen Recht, 1942, S. 129. 
soweit konkretisierend der Verweis auf das Volk. Das Volk als eine vorgegebene, gedachte, aber auch real erfahrbare, durch Sprache, Geschichte, Kultur allmählich gewachsene, Orientierung stiftende und Heimat vermittelnde Größe. Auf dieser Grundlage kann Demokratie als die Selbstbestimmung des Kollektivs „Volk“ begriffen und real erfahrbar werden. Die Bürgerschaft in der antiken Polis praktizierte in diesem Sinne „demokratische Selbstbestimmung" ebenso wie die demokratischen Bestrebungen im 19. Jahrhundert, in denen das Volk nach und nach zum nicht mehr abzuweisenden politischen Faktor wurde und sich anschickte, sich selbst regieren zu wollen. Diese Idee der „Demokratie“ ist eine aus der Geschichte der Staatsformenlehre gegenwärtig und auch in Zukunft nicht hinwegzudenkende Legitimationskategorie politischer Herrschaft.

\section{Die Geburt der Demokratie im Schoße des Nationalstaates}

In eher statischen Zeiten war der unscharfe Begriff des Volkes als Chiffre einer gegebenen Gemeinschaft im Prinzip kaum umstritten. Das änderte sich, als sich allmählich die Idee der Menschenrechte entwickelte und sich der Volksbegriff in Gegenbewegung dazu rechtfertigen mußte. Denn die Idee der Menschenrechte kennt nur ebenso abstrakte wie individuelle Menschen, aber keine kollektive Einheiten von Menschen. So entstanden im 19. Jahrhundert die manigfaltigen Narrative der europäische Völker und grundierten derart den aufkommenden Nationalismus. Dieses erzählte „Volk“ war zwar niemals das, was der Nationalismus des 19. Jahrhundert daraus gemacht hatte. Stets war „Volk“ ein Sammelbegriff für ein sich ständig wandelndes, durch $\mathrm{Zu}$ - und Abwanderung, durch Eroberungen oder Gebietsverluste sich veränderndes Substrat. Aber über alle Kontinuitätsbrüche und Wandlungsprozesse hinweg bezeichnete das Volk stets ein gegebenes Substrat einander - mehr oder weniger, tatsächlich oder nur eingebildet - vertrauter Menschen. Mit und seit der französischen Revolution wurde dieses Volk auch als politische Größe immer wieder real als „verfassunggebende Gewalt“ wirksam: „Wir sind das Volk“ skandierten die Bürger der DDR und erzwangen die politische Einheit Deutschlands. Und diese zwar im einzelnen undeutliche, aber real vorhandene Größe „Volk“ wurde dann über das Recht der Staatsangehörigkeit rechtlich in Form gebracht, formalisiert. Auf den Punkt gebracht: die neuzeitliche Geburt der Demokratie entstand aus dem Geiste und im Schoße des National- 
staates. ${ }^{8}$ Demokratie ist Selbstbestimmung von Völkern, d.h. nur einer Teilmenge der gesamten Menschheit, nicht Teilhabe beliebiger, zufällig und zeitweise räumlich anwesender Menschen. Diese Konnektivität wird durch zwei Entwicklungen faktischer Art immer mehr in Frage gestellt: den Prozeß der Individualisierung und weltweite Migrationsbewegungen.

\section{Auflösung des „Volkes“ durch Individualisierung}

Unter Individualisierungsdruck verdunstet gegenwärtig in den westlichen Gesellschaften immer mehr die Vitalität von prinzipiell vorgegebenen und lebenslang bindenden Gemeinschaften. Schon 1887 diagnosizierte Ferdinand Tönnies hellsichtig die sich abzeichnende Schwächung von Gemeinschaften - staatlichen, religiösen, familiären - und ihre Ersetzung durch zweckorientierte Gesellschaften und selbstbestimmte Zusammenschlüssen von Individuen zu bestimmten Zwecken und auf selbstbestimmte Zeit. ${ }^{9}$ Im Ergebnis unterliegen die Menschen heute den sich teils widersprechenden Logiken unterschiedlicher Systeme, von denen das politische System nur eines unter vielen ist. Aber auch innerhalb der Systeme wird heute stete Veränderungsbereitschaft erwartet und gelebt: wenn Projekte gelaufen sind, der Zweck erfüllt, zerfällt der Zusammenschluß, um sich gegebenenfalls anderweitig mit anderen Partnern mit anderen Zielen neu zu formieren. Dieser Entwicklung zu einer hochgradig ausdifferenzierten und maximal flexiblen Gesellschaft eröffnet dem Einzelnen historisch unvergleichliche Handlungsoptionen: wenn das Individuum scheitert oder es neue Präferenzen setzt, wechselt es den Handlungszusammenhang: den Partner, den Verein, den Staat, die Religion. Pate hinter dieser Entwicklung steht ein die Gegenwart prägender Zeitgeist: der moderne Mensch scheut sich, seine individuelle Freiheit in verbindliche Bindungen zu überführen, sondern ist bestrebt, sich möglichst alle Optionen so lange als möglich offenhalten. Daher das Sterben der Vereine, der Rückgang der Parteimitglieder, die Marginalisierung der Ehe, die begehrte Mehrstaatsangehörigkeit, um nur einige Felder zu nennen. An ihre Stelle treten Projekte, Bindungen und Partnerschaften auf Zeit, Optionsangebote wie Schnupperparteimitglied-

8 Vgl. dazu näher Depenheuer, Fn. 2, S. 39.

9 Vgl. Ferdinand Tönnies, Gemeinschaft und Gesellschaft, 1887; Norbert Elias, Die Gesellschaft der Individuen, 2001; Markus Schroer, Das Individuum der Gesellschaft, 2001. 
schaften in Parteien oder optionale Staatsangehörigkeiten etc. Unterfüttert und befeuert wird dieser Wandlungsprozeß durch die umfassende Digitalisierung aller Lebensbereiche, die den Einzelnen scheinbar souverän stellt. Dieser Prozeß der Individualisierung stellt an die Idee der demokratisch organisierten Repräsentation des Volkes eine existenzielle Frage: kann eine Summe von Individuen überhaupt repräsentiert werden? Steht das Selbstverständnis der Individuen der Idee der Repräsentation eines Kollektivs nicht fundamental entgegen? Kann eine beliebige Ansammlung von Menschen repräsentiert werden? Oder läßt sich in einer Gesellschaft von Individuen doch noch ein gemeinsames Sein erkennen, das sich repräsentieren ließe?

\section{Auflösung des „Volkes“ durch Migration?}

Der ursprüngliche Gleichklang von Volk und Demokratie wird im Zeitalter von Globalisierung und Migration noch in einer weiteren Hinsicht, diesmal gleichsam „,von außen“, immer fragwürdiger. Deutschland hat sich faktisch zu einem Einwanderungsland entwickelt. Ausgehend von früher ungeahnten Reisemöglichkeiten über diverse Wellen des Zuzugs von Gastarbeitern bis hin zu weltweiten Wanderungsbewegungen in erheblichem Umfang verfügen in Deutschland gegenwärtig 20\% der Gesamtbevölkerung über einen Migrationshintergrund. In manchen Stadtteilen und Schulen liegt der Ausländeranteil bei über 50\%. Zwei Drittel der ausländischen Bevölkerung leben seit über 10 Jahren in Deutschland, nahezu $40 \%$ seit über 20 Jahren und ein Viertel seit über 30 Jahren. ${ }^{10}$ Ebenso wie Individualisierungs- lassen sich auch Migrationsprozesse als Ausdrucksformen der Individualisierungsschübe moderner Gesellschaften verstehen: denn es wandern nicht Völker, sondern Menschen, nicht Kollektive, sondern Individuen. Jedenfalls bezeichnet dieser Befund ein demokratietheoretisches Problem. Was also wird aus der „Herrschaft des Volkes“, wenn das Substrat des Volkes ,undeutlich“ wird?

Nun haben Juristen eine symphatische Scheu, sich mit der Wirklichkeit auseinander setzen zu müssen. Ihr Hoheitsgebiet ist das Reich des Sollens, das sich in Normen zeigt. Deshalb können sie die Frage, was das Volk so-

10 Einzelheiten: Bundesamt für Migration und Flüchtlinge, Migrationsbericht 2010, S. $179 \mathrm{f}$. 
ziologisch ist, dadurch umgehen, daß sie rechtlich definieren, was das Volk ist. Ergebnis: „Deutscher im Sinne dieses Gesetzes ist, wer die deutsche Staatsangehörigkeit besitzt" ( 1 1 StAG). Aber dadurch wird das dahinterstehende Problem nicht gelöst, sondern nur dissimuliert. Denn die Frage lautet: sollen alle, die faktisch da sind, auch rechtlich dazugehören? Kann man also auf das Institut der Staatsangehörigkeit als Voraussetzung der demokratischen Staatsform verzichten? Immerhin sind beide Entwicklungen - Individualisierung innerhalb der staatlichen Gemeinschaft, weltweite grenzenüberschreitende Migrationsbewegungen andererseits - faktische Gegebenheiten, die überkommene Rechtsvorstellungen und -institute in Frage stellen. Gerade deshalb bilden sie eine ernsthafte Herausforderung für die Zukunft der repräsentativen Demokratie in der Gegenwart.

In Deutschland besteht insoweit die Neigung, die Probleme wohlwollend zu marginalisieren und diese Entwicklungen als Nebenwirkungen einer politisch erfreulichen Zeit $\mathrm{zu}$ interpretieren, einer entpolitisierten Zeit, in der es - spätestens seit dem Zusammenbruch der kommunistischen Staaten - keine wirklich politischen Probleme mehr zu geben scheint: aus Staatsbürgern werden Menschen, aus kulturellen Unterschieden erwächst eine „bunte Gesellschaft“, aus Monokulti wird Multikulti. Tatsächlich entfalteten sich Individualisierung und Zuwanderung in Deutschland im Kontext und als Folge einer hochgradig entpolitisierten Wirklichkeit. Deutschland war militärisch besiegt, durch die Alliierten besetzt, in zwei Staaten geteilt. Es verfügte über keine Souveränität, stand aber unter dem Schutzschirm der NATO. Derart vor politischen Fährnissen weithin behütet entwickelte es sich zu einer Hochburg des angenehmen, ethisch allzeit korrekten, unpolitischen Lebens: immer mehr Wohlstand im „Wirtschaftswunderland“, immer mehr Freiheitsräume für den Einzelnen, immer sensiblere Deutungen der „unantastbaren Menschenwürde“, immer rechtsstaatlicher und freiheitsfreundlicher, immer friedlicher, immer sozialer, immer internationaler, immer offener, immer inklusiver, immer bunter - Leben im politischen Schlaraffenland. Doch diese schönen unpolitischen Zeiten dürften allmählich ihrem Ende entgegengehen. Und damit könnten auch jene Bedingungen entfallen, die die Entpolitisierung der Gesellschaft ermöglichten. Dann könnte auch das von vielen vernachlässigte Problem der politischen Dimension des Volkes wieder zum Thema werden. 


\section{Die politische Dimension des Volkes}

Es gibt noch Staaten auf diesem Globus. Die Feststellung klingt trivial, zeitigt aber Konsequenzen: solange es nämlich eine Vielzahl von Staaten gibt - und das dürfte allen Tendenzen zur Bildung von Staatengruppierungen zum Trotz noch einige Zeit so bleiben -, solange wird es auch Politik geben. ${ }^{11}$ Politik bezeichnet bekanntlich die Möglichkeit, ja die Wahrscheinlichkeit von Interessengegensätzen zwischen Staaten und Staatengruppen, mögen sie Umwelt- und Energiefragen, wirtschaftliche oder politische Einflußsphären betreffen. Daraus entspringen Interessengegensätze, die zu Rivalitäten und Koalitionen, d.h. zu „Machtfragen“ werden können. Diese können bekanntlich friedlich durch Verhandlungen oder Schiedsentscheide zu einem (vorübergehenden) Arrangement auf Zeit oder aber auch unfriedlich bis hin zu bewaffneten Konflikten einer Lösung zugeführt werden. Ich verzichte darauf, die gegenwärtig (2015) zahlreichen aktuellen bewaffneten Konflikte aufzuzählen und die divergierenden - offenen wie latenten - Interessenlagen zu skizzieren, die zu weiteren Konfliken führen könnten. Noch wähnen sich viele in Deutschland außerhalb dieser Konflikte. Aber das kann sich schneller ändern als uns das lieb ist. Entscheidend aber ist hier nur, daß es diese Konflikte latent immer gibt und sie aus gegebenem Anlaß auch militärisch eskalieren können, sich zur Unterscheidung von Freund und Feind verdichten. ${ }^{12}$ Und weil es diese politischen Gegensätze zwischen Staaten gibt, unterscheiden wir rechtlich wie faktisch zwischen Staaten und zwischen den Bürgern dieser Staaten: sie befinden sich entweder auf der einen oder anderen Seite. In derartigen Ausnahmefällen wird jeder - nolens volens - Partei und dann stellt sich die Frage nach der Loyalität der Bevölkerung: steht sie hinter dem eigenen Staat oder nicht.

\section{Funktion und Bedeutung der Staatsangehörigkeit}

Eine Antwort darauf könnte das Institut der Staatsangehörigkeit sein. Die rechtlich definierte Staatsangehörigkeit bezeichnet die formalisierte Basis des Bürgerstatus und bedeutet politische Vollmitgliedschaft im Gemein-

11 Politik im Sinne von Carl Schmitt, Der Begriff des Politischen [1928], 1963, S. $20 \mathrm{ff}$.

12 Ebenso klassische wie umstrittene Begriffsbildung Schmitt, Fn. 11, S. 26 ff. 
wesen. ${ }^{13}$ Sie bildet die Grundlage gleicher politischer Teilhabe aller Bürger bei der politischen Willensbildung und der dadurch demokratisch legitimierten Kreation der Staatsorgane durch Wahlen und Abstimmungen (Art. 20 Abs. 2 GG). Mit den Worten des Bundesverfassungsgerichts: „Die Staatsangehörigkeit ist die rechtliche Voraussetzung für den gleichen staatsbürgerlichen Status, der einerseits gleiche Pflichten, zum anderen und vor allem aber auch die Rechte begründet, durch deren Gewährleistung die Staatsgewalt in der Demokratie legitimiert wird." ${ }^{14}$ Dieser formal-demokratische Bürgerbegriff öffnet den Zugang zu den politischen Institutionen und demokratischen Verfahren, um die Art, Form und Regeln des politischen Zusammenlebens demokratisch zu gestalten und zu verändern. Solange die Staaten primärer Bezugspunkt demokratischer Herrschaftsausübung sind, ist die konstitutive Bedeutung der Staatsangehörigkeit für die demokratische Herrschaftsform ebenso unvermeidbar wie die dadurch bewirkte Unterscheidung zwischen Staatszugehörigen und Fremden.

Die rechtliche Bedeutung der Staatsangehörigkeit erschließt sich indes nicht aus dem Status als solchem, sondern aus den Rechtsfolgen, die an die Staatsangehörigkeit anknüpfen. Insoweit werden wir gegenwärtig Zeugen einer weitgehenden rechtlichen Marginalisierung der Staatsbürgerschaft als exklusivem Statusrecht. Scheinbar unaufhaltsam wird der tatsächliche Rechtsstatus von Staatsangehörigen und Ausländern immer gleicher. ${ }^{15}$ Ein kursorischer Überblick mag diesen Befund illustrieren: das Aufenthaltsrecht ist heute praktisch kein exklusives Privileg der Staatsangehörigen mehr. Aufenthaltsentscheidungen werden unions-, grund- und menschenrechtlich überlagert. Unionsbürgern wird qua Binnenfreizügigkeit ein Recht auf Aufenthalt garantiert. Mit der Gewährung von Gebietszugang eines jeden Ausländers verfestigt sich dessen Status im Aufenthalt. Der ursprünglich als vorübergehend konstruierte Aufenthaltsstatus geht über in ein Bündel von Aufenthaltstiteln, die der Tendenz nach zu einem

13 Einzelheiten: Stefan Haack, Staatsangehörigkeit - Unionsbürgerschaft - Völkerrechtssubjektivität, in: J. Isensee/P. Kirchhof (Hrsg.), Handbuch des Staatsrechts, Deutschland in der Staatengemeinschaft, Bd. X, 3. Aufl., 2012, § 205, Rn. 7 ff.

14 BVerfGE 113, 27 (294).

15 Zum Folgenden jeweils m.w.N. Christian Walter und Klaus Ferdinand Gärditz, Der Bürgerstatus im Licht von Migration und europäischer Einwanderung, in: VVDStRL 72 (2013), S. 7 ff., 49 ff. 
rechtlich gesicherten Daueraufenthalt führen können: das Aufenthaltsrecht des Ausländers kann gleichsam „durch Ersitzung“ erworben werden.

Aber auch der Status des rechtmäßig in Deutschland verweilenden Ausländers wird dem der deutschen Staatsangehörigen immer ähnlicher: die Gleichstellung von In- und Ausländern als Wirtschaftssubjekte ist durchgehend verwirklicht, für Unionsbürger bildet sie gar die Essenz der Grundfreiheiten. Allein der Arbeitsmarktzugang für drittstaatsangehörige Ausländer ist noch reguliert. Der Zugang zum öffentlichen Dienst ist für Unionsbürger in weitem Umfang geöffnet. Nur Justiz, Diplomatie und innere Sicherheit bilden letzte Reservate, die exklusiv Staatsangehörigen vorbehalten sind, wenngleich auch hier erste Öffnungen zu beobachten sind. Im übrigen unterliegen In- wie Ausländer in gleicher Weise der allgemeinen Gehorsamspflicht gegenüber geltendem Recht, haben beide Gruppen gleichen Anspruch auf soziale Teilhabe: Leistungen werden einfachgesetzlich entweder nach Versicherungsprinzip oder nach Bedürftigkeit organisiert, in der Regel jedoch nicht nach Staatsangehörigkeit. Elementare Sozialleistungsansprüche sichern das in der Menschenwürde wurzelnde und damit das statusunabhängige soziale Existenzminimum: Mensch ist nämlich jeder. So hat das Bundesverfassungsgericht jüngst den Ausschluss ausländischer Staatsangehöriger mit nur vorübergehenden, humanitär motivierten Aufenthaltstiteln von Bundeserziehungs- und Bundeselterngeld für gleichheitswidrig erklärt: wer rechtmäßig in Deutschland verweilt, bekommt sozialstaatlich das der Menschenwürde je gemäße. ${ }^{16}$ Auch die staatliche Schutzverpflichtung erfaßt alle - Staatsangehörige wie Fremde - gleichermaßen: eine Differenzierung nach der Staatsangehörigkeit ist grundrechtlich zwar bei Deutschengrundrechten zulässig, wird jedoch einfachrechtlich regelmäßig neutralisiert.

Fazit: Die festzustellende allmähliche Annäherung des Status von Inund Ausländern - überlagert von der Differenzierung zwischen Unionsbürgern sowie sonstigen privilegierten Drittstaatsangehörigen - bewirkt eine Auffächerung der vormals exklusiv durch die Staatsangehörigkeit vermittelten Statusverhältnisse. Die Bedeutung des Staatsangehörigkeitsstatus nimmt korrespondierend ab. Nicht zufällig nehmen gleichzeitig entsprechend mehrfache Staatsangehörigkeiten zu. Unverfügbarkeit und Unentrinnbarkeit des Staatsangehörigkeitsbandes verlieren somit immer mehr an Bedeutung. Eine Bestandsaufnahme der Rechtsentwicklung zeigt ein-

16 BVerfG, NVwZ 2012, S. 1024. 
mal mehr: Statusunterschiede zwischen In- und Ausländern verblassen mit Verfestigung des Aufenthalts und werden in der Folge zu Details des Verwaltungsrechts, das die aus den Uneindeutigkeiten der rechtlichen Zugehörigkeiten entstehenden Probleme im Einzelfall abarbeiten muß. Wenn also die aus der Staatsangehörigkeit fließenden Rechte mit denen immer ähnlicher werden, die aus dem rechtmäßigen Aufenthalt von Ausländern im Bundesgebiet resultieren, dann stellt sich unvermeidlich die weitergehende Frage, welche Konsequenzen daraus für das Wahlrecht folgen. Noch markiert das demokratische Wahlrecht den entscheidenden Statusunterschied zwischen Staatsangehörigen und Ausländern. Aber kann das so bleiben oder müssen nicht vielmehr alle Ausländer, insbesondere wenn sie längere Zeit im Bundesgebiet ansässig sind, Steuern zahlen, im sozialen Leben integriert sind, dann nicht auch demokratisch am politischen Gemeinwesen partizipieren dürfen? Haben sie nicht gar einen menschenrechtlichen Anspruch auf demokratische Teilhabe? Ist ihre demokratische Exklusion ein verfassungsrechtliches Relikt und deswegen überholt?

\section{E. Demokratische Exklusion von Ausländern als Verfassungsgebot}

Nimmt man das positive Verfassungsrecht zur Grundlage für die Beantwortung dieser Fragen, dann ist der Befund eindeutig. Im Hinblick auf demokratische Teilhabe und Repräsentation besteht die fundamentale Statusdifferenz zwischen Staatsangehörigen und Ausländern bis heute verfassungsrechtlich fort. Das Volk, von dem alle Staatsgewalt ausgeht (Art. 20 Abs. 2 Satz 1 GG), ist die Summe der Staatsangehörigen, die insoweit das demokratische Zurechnungs- und Legitimationssubjekt konstituieren und Herrschaftslegitimation personalisieren. In der Konsequenz dieser rechtlichen Vorgaben bleibt Ausländern das aktive Wahlrecht zu Landesparlamenten und zum Deutschen Bundestag, das Herzstück des status activus, verschlossen. In den Worten des Bundesverfassungsgerichts aus dem Jahre 1990: „Der Verfassungssatz ,Alle Staatsgewalt geht vom Volke aus“ enthält [...] nicht allein den Grundsatz der Volkssouveränität. Vielmehr bestimmt diese Vorschrift selbst, wer das Volk ist, das in Wahlen, Abstimmungen und durch besondere Organe der Gesetzgebung, der vollziehenden Gewalt und der Rechtsprechung Staatsgewalt ausübt: Es ist das Staatsvolk der Bundesrepublik Deutschland. [...] Als demokratischer Staat kann sie nicht ohne die Personengesamtheit gedacht werden, die Träger und Subjekt der in ihr und durch ihre Organe ausgeübten Staatsgewalt 
ist. Diese Personengesamtheit bildet das Staatsvolk, von dem alle Staatsgewalt ausgeht. Art. 20 Abs. 2 Satz 1 GG hat daher nicht zum Inhalt, daß sich die Entscheidungen der Staatsgewalt von den jeweils Betroffenen her zu legitimieren haben; vielmehr muß die Staatsgewalt das Volk als eine zur Einheit verbundene Gruppe von Menschen zu ihrem Subjekt haben. Das Volk, von dem die Staatsgewalt in der Bundesrepublik Deutschland ausgeht, wird nach dem Grundgesetz von den deutschen Staatsangehörigen und den ihnen nach Art. 116 Abs. 1 gleichgestellten Personen gebildet. Die Zugehörigkeit zum Staatsvolk der Bundesrepublik wird also grundsätzlich durch die Staatsangehörigkeit vermittelt. Die Staatsangehörigkeit ist die rechtliche Voraussetzung für den gleichen staatsbürgerlichen Status, der einerseits gleiche Pflichten, zum anderen und insbesondere aber auch die Rechte begründet, durch deren Ausübung die Staatsgewalt in der Demokratie ihre Legitimation erfährt. [...] Schließlich weist Art. 146 dem deutschen Volke die Entscheidung über eine das Grundgesetz zu gegebener Zeit ablösende Verfassung zu. In nicht zu übersehender Parallelität erklären die Präambel und Art. 146 GG das deutsche Volk zum Träger und Subjekt des Staates der Bundesrepublik Deutschland. [...] Ist also die Eigenschaft als Deutscher nach der Konzeption des Grundgesetzes der Anknüpfungspunkt für die Zugehörigkeit zum Volk als dem Träger der Staatsgewalt, so wird auch für das Wahlrecht, durch dessen Ausübung das Volk in erster Linie die ihm zukommende Staatsgewalt wahrnimmt, diese Eigenschaft vorausgesetzt." 17

Die Einführung eines allgemeinen Ausländerwahlrechts ist auf der Grundlage des geltenden Verfassungsrechts in seiner maßgeblichen Interpretation durch das Bundesverfassungsgericht also unzulässig. Das schließt andere Formen der Ausländerpartizipation (Ausländerbeiräte etc.) nicht aus; aber diese sind kein Ausdruck demokratischer Teilhabe und deshalb auch nicht geeignet, Entscheidungen demokratisch zu legitimieren. Die Staatsangehörigkeit entfaltet damit für die Teilhabe an der demokratischen Staatswillensbildung nach wie vor eine verfassungsrechtlich vorgegebene und politisch zu respektierende Exklusionswirkung.

17 BVerfGE 83, 37, 50 ff. - Ausländerwahlrecht. 


\section{F. Demokratische Inklusion von Ausländern als politische Herausforderung}

Dieser verfassungsrechtliche Befund bereitet vielfach rechtspolitisches Unbehagen. Als bedeutende Exportnation stehe das wohlhabende Deutschland im Zentrum des Prozesses von Globalisierung und Internationalisierung, von Migrationsbewegungen und Integrationsprozessen. Dadurch entwickelten sich die der Herrschaftsgewalt Unterworfenen und die Mitglieder des Legitimationssubjekts Volk auseinander. Die im Bundesgebiet wohnhaften Ausländer verfügten über kein Wahlrecht, seien nicht Mitglied der politischen Gemeinschaft. Ihre Anliegen seien nicht die eines Wählers und würden deshalb auch nur verzerrt oder gar nicht berücksichtigt. Obwohl sie die Lasten von Herrschaft mittrügen, seien sie von politischer Mitbestimmung ausgeschlossen. Der dauerhafte Aufenthalt von Ausländern ohne Wahlrecht wird derart als schwärendes Partizipationsproblem wahrgenommen. Wer Steuern und Abgaben zahle, müsse auch politisch mitbestimmen dürfen. Die Parole des amerikanischen Unabhängigkeitskrieges „No Taxation without Representation“ verdeutliche die politische Sprengkraft dieses Korrelationsverhältnisses. Aus diesen Überlegungen wird dann die rechtspolitische Forderung nach verfassungsrechtlicher Inklusion von in Deutschland lebenden Ausländern abgeleitet. Eine repräsentative Begründung liest sich wie folgt: ${ }^{18}$ Die unbestrittene Entwicklung, daß Ausländer zahlreiche Möglichkeiten haben, einzelne oder gar einen großen Teil der mit der Staatsbürgerschaft üblicherweise verbundenen Statusrechte zu erwerben, ,entbündele“ die Statusrechte. Die materiellen Rechte der Staatsbürgerschaft, insbesondere ein dauerhaftes Aufenthaltsrecht, würden weitgehend unabhängig von der Staatsangehörigkeit zugewiesen. Dies führe dazu, daß sich Migranten mit dauerhaftem Aufenthaltsrecht hinsichtlich des Bürgerstatus in der Praxis letztlich nur noch beim Wahlrecht von den Staatsangehörigen unterscheiden. Solange die Statusrechte überwiegend gebündelt mit dem Bürgerstatus verliehen wurden, hätte der Bürgerstatus Gleichheit auch hinsichtlich der Partizipationsmöglichkeiten garantieren können. In dem Maße, in dem der Bürgerstatus entbündelt und der dauerhafte Aufenthalt unabhängig von der Staatsangehörigkeit ermöglicht werde, drohten Ungleichheiten, die sich als demokratisches Mitwirkungsproblem darstellten. Für die repräsentative Demokra-

18 Walter, Fn. 15, S. 32 f.

https://doi.org/10.5771/9783845274072-201, am 26.04.2023, 16:18:17 
tie könne das zum Krisenfall werden, denn es drohe eine Inkongruenz „Zwischen den Inhabern demokratischer politischer Rechte und den dauerhaft einer bestimmten staatlichen Herrschaft Unterworfenen“".

Das hier verwandte Argumentationsmuster ist bekannt: eine Entwicklung wird festgestellt (Einwanderung), die Richtung ihrer rechtlichen Bewältigung analysiert (Entkopplung von Statusrechten und Staatsangehörigkeit), die weitere Entwicklung interpoliert und hochgerechnet (Funktionslosigkeit des Staatsangehörigkeitsrechts), schließlich der status futurus skizziert (Ablösung des Wahlrechts von der Staatsangehörigkeit), dessen ohnehin zu erwartender Eintritt dann auch schon hier und heute als ,historisch zwingend" politisch und rechtlich vorweggenommen werden könne (demokratische Partizipation). Allenfalls über die rechtstechnische Umsetzung des als überfällig Erkannten kann dann noch diskutiert werden. So werden für die rechtspolitisch erwünschte demokratische Inklusion von Ausländern denn auch mehrere Lösungswege angeboten. Der erste hält rechtstechnisch an der Statuslösung fest, setzt aber auf erleichterte Einbürgerung, gegebenenfalls auch unter vermehrter Hinnahme von doppelten Staatsangehörgkeiten. Alternativ soll das Wahlrecht von der Staatsangehörigkeit abgekoppelt, der Prozeß der Entbündelung der Statusrechte vollendet und das Wahlrecht für die Bevölkerung, also jeden im Inland lebenden Ausländer eingeführt werden, um derart Deutschland endlich wieder demokratisch werden zu lassen. Ich beschränke mich auf letzteren Vorschlag. ${ }^{19}$

\section{G. Allgemeines Wahlrecht der „Bevölkerung“?}

Der Vorschlag, die schon weit fortgeschrittene Entbündelung des Bürgerstatus endlich zu vollenden und auch das Wahlrecht nicht mehr an den Status, sondern an den dauerhaften Aufenthalt zu knüpfen, ${ }^{20}$ wird trotz der insoweit deutlichen Absage des Bundesverfassungsgerichts auf der rechtspolitischen Tagesordnung gehalten. Diese Tatsache ist in Ansehung der Möglichkeit erleichterten Erwerbs der deutschen Staatsangehörigkeit mehr als nur erstaunlich. Zwar wurde von der erweiterten Möglichkeit der Ein-

$19 \mathrm{Zu}$ den anderen Strategien vgl. Depenheuer, Fn. 2, S. 46 ff.

20 Vgl. etwa Walter, Fn. 15, S. 37 ff. 
bürgerung nur in geringem Umfang Gebrauch gemacht, ${ }^{21}$ das zentrale Ziel der Reform, dem Auseinanderfallen von Wohnbevölkerung und Staatsbürgern durch erweiterte Einbürgerungsmöglichkeiten entgegenzutreten, aber nicht erreicht. Doch muß man dies nicht als Ausdruck einer freien Entscheidung der adressierten Ausländer in Ansehung einer rechtlichen Option interpretieren? Zeigt sich in der Nichtwahrnehmung der Einbürgerungsoption, daß die betreffenden Ausländer offensichtlich keinen erhöhten Wert darauf legen, Deutsche zu werden und demokratische Partizipationsrechte wahrzunehmen? Könnte man dies nicht auch politisch akzeptieren, statt ihnen ein fortgesetzes Leiden an fehlenden politischen Partizipationsproblemen in ihrem Wohnsitzland anzudichten? Wer die deutsche Staatsangehörigkeit erwerben könnte, die Option aber nicht ergreift, wird dafür sicherlich seine wohlerwogenen Gründe haben. Vielleicht spüren oder wissen die betreffenden Ausländer besser als die Verfechter einer staatsangehörgkeitsrechtlichen Inklusion, daß hinter der Entscheidung für oder gegen eine Staatsangehörigkeit potentielle Loyalitätskonflikte stehen, die auch einmal zum Schwur kommen könnten. Daß niemand Diener zweier Herren sein kann, ist nicht nur uralte, schon im Matthäus-Evangelium bezeugte Wahrheit und Weisheit. ${ }^{22}$ Und die Staatsangehörigkeit bezeichnet eine politische Loyalitätsbindung, deren Potential nicht in Sonnenscheinzeiten, sondern in Konfliktlagen sichtbar, relevant und entscheidend werden kann. Welches Konfliktpotential sich dabei aufbauen und entladen kann, zeigte mehr als deutlich der Aufruf des türkischen Ministerpräsidenten, der 2008 in Köln und in Gegenwart des deutschen Innenministers an „seine deutschen Landsleute“, die die deutsche Staatsangehörigkeit angenommen haben: „Assimilierung ist ein Verbrechen gegen die Menschlichkeit". Die in Deutschland lebenden Türken sollten auch als Staatsbürger anderer Länder zuerst ihre Muttersprache lernen und ihre Kultur beibehalten. Hier wird das Dilemma doppelter Loyalitäten in nuce erkennbar. Im Ernstfall, d.h. wenn sich die zwei Herren mal in die Haare bekommen, wird die Tiefenstruktur der Staatsangehörigkeit sichtbar: sie bezeichnet diejenige politische Gemeinschaftsbindung, der man sich im

21 Der politisch erwartete Erfolg der Reform hielt sich in Grenzen. Die Quote der Gesamtzahl der eingebürgerten Ausländer in Deutschland beläuft sich insgesamt auf 2,44\%, bei türkischen Staatsangehörigen auf 2,0\%. Vgl. Walter, Fn. 15, S. 33 ff. m.w.N.

22 Matthäus 6, 24. 
existenziellen Grenzfall verbunden weiß.23 Und dann heißt die Alternative: entweder man ist Türke in Deutschland oder Deutscher türkischer Abstammung. Tertium non datur. Es entspräche daher freiheitlicher Tugend und demokratischer Toleranz, die Entscheidung der adressierten Ausländer gegen den Erwerb der deutschen Staatsangehörigkeit schlicht zu respektieren und zu akzeptieren. Doch die Freunde der Inklusion sind auf die Einführung eines Ausländerwahlrechts fixiert.

Verfassungsrechtliches Vehikel dieser partizipativen Zwangsbeglückung soll nunmehr die Menschenwürde des Grundgesetzes sein. Tatsächlich hat das Bundesverfassungsgericht in seiner Lissabon-Entscheidung einen engen Bezug zwischen Demokratie und Menschenwürde hergestellt: „Der Anspruch auf freie und gleiche Teilhabe an der öffentlichen Gewalt ist in der Würde des Menschen (Art. 1 Abs. 1 GG) verankert." 24 Doch ist es kühn, von dieser Sentenz auf eine Relativierung, wenn nicht Überwindung der Vorstellung zu schließen, nach der es einer vorrechtlich geprägten Homogenität unter einer Gruppe von Menschen nicht mehr bedarf, um sich im Zeichen der Demokratie zu einer politischen Einheit zusammenzuschließen. ${ }^{25}$ Denn die öffentliche Gewalt, von der das Gericht in diesem Zusammenhang spricht, ist die durch das Grundgesetz konstituierte, also diejenige, die sich - nach der Präambel - das deutsche Volk gegeben hat. Die grundgesetzliche Menschenwürde gegen die vom Deutschen Volk konstituierte Staatlichkeit, gegen die vom Deutschen Volk sich gegebene Verfassung und gegen die verfassungsrechtlich normierte Selbstbestimmung des Deutschen Volkes auszuspielen, ist ein schlechter verfassungsrechtlicher Taschenspielertrick. Daher geht die auf diesen Satz aufbauende Forderung in elementarer Weise fehl: „Die zentrale Bedeutung der Menschenwürde für die deutsche Verfassungsordnung müsse auch zu einer

\section{Vgl. Schmitt, Fn. 11, S. 26 ff.}

24 BVerfGE 123, 267 (341). Kritisch gegen einen apriorischen Gleichklang von Demokratie und Menschenrechten Ernst-Wolfgang Böckenförde, Das Unwahrscheinliche wollen, in: FAZ v. 2.5.1996, S. N 6.

25 Vgl. Josef Isensee, Nachwort zu: Europa - die politische Erfindung eines Erdteils, in: J. Isensee (Hg.), Europa als politische Idee und als rechtliche Form, 2. Aufl. 1994, S. 122: „Ohne ein gewisses Maß an Homogenität kann kein Staat bestehen. Der Wille zur politischen Einheit, der eine Menschengruppe zum Volk als Nation und damit zum möglichen Subjekt demokratischer Selbstbestimmung werden läßt, knüpft an objektive Vorgegebenheiten an, etwa geopolitische Lage, wirtschaftliche Interessen, Geschichte, Sprache, zivilisatorische Standards, Ethos, Kultur, Religion.". 
großzügigeren Herangehensweise an die Interpretation des Volksbegriffs in Art. 20 Abs. 2 GG führen. ${ }^{26}$ So wird Verfassungsinterpretation zum Modus einer rechtspolitisch erwünschten großzügigen Sozial- und Integrationspolitik.

\section{H. Demokratie ohne Demos - Auf dem Weg in die Fremdherrschaft?}

Gegenüber derartigen Uminterpretationen des Grundgesetzes gilt es als normativen Bestand festzuhalten: solange „das Volk sich als politisches Herrschaftssubjekt nicht nach dem Menschsein schlechthin, sondern nach selbst geschaffenen normativen Kriterien ein[setzt]", bedeutet das reziprok immer auch „den Ausschluss derjenigen von der politischen Herrschaft, die nicht Bestandteil des durch demokratisches Recht definierten Volkes sind“. ${ }^{27}$ Die Menschenwürdegarantie des Grundgesetzes entfaltet sich auf der Grundlage politischer Einheit, nicht umgekehrt. Die Demokratie des Grundgesetzes ist somit konkretisierte Menschenwürde, indem sie die Identität von Herrschern und Beherrschten etabliert und einen realen Verantwortungszusammenhang statuiert, der durch regelmäßige demokratische Wahlen etabliert und realisiert wird. Nur dann liegt „ein Verantwortungszusammenhang der wirklichen, ernsten Art [sc. vor], in dem nicht nur Worte zu wechseln, sondern auch, von Wählern wie Gewählten, Konsequenzen des eigenen Entscheidungsverhaltens zu tragen sind." 28 Solange es ein Pluriversum von Staaten gibt, gibt es Politik, gibt es politisch zu unterscheidende Völker, zu denen man gehört oder nicht gehört, gibt es die Unterscheidung demokratischer Selbstbestimmung oder undemokratischer Fremdbestimmung. Staatlichkeit als politische Schicksalsgemeinschaft läßt sich nicht menschenrechtlich unterlaufen: Mensch wird der Mensch nur in politisch kontingenter Gemeinschaft. ${ }^{29}$

Das einzigartige politische Experiment der Europäischen Union könnte hier ein neues Kapitel der Geschichte der Demokratie schreiben. Die europäischen Staaten der Gegenwart zielen auf eine wirtschaftliche und

26 So Walter, Fn. 15, S. 39 f.

27 K.F. Gärditz/C. Hillgruber, Volkssouveränität und Demokratie ernst genommen Zum Lissabon-Urteil des BVerfG, JZ 2009, 872 ff. (873).

28 Lübbe-Wolf, abw. Meinung BVerfG, NVwZ 2012, 1167 (1173).

29 Grundsätzlich zum Problem: Panajotis Kondylis, Planetarische Politik nach dem Kalten Krieg, 1992, S. 114 ff. 
politische Union, öffnen sich gegenüber Staaten und Bürgern anderer Staaten. Gleichwohl bewahren sie ihre Eigenständigkeit in einem durch Geburt und Herkunft verwandten Staatsvolk, einem ihm zugehörigen Raum und der kulturellen Gemeinsamkeit von Sprache, Religion, Kunst und geschichtlicher Erfahrung. Die dadurch unvermeidlich bewirkte Erosion demokratischer Substanz bei den Mitgliedsstaaten ist ebenso unübersehbar wie ein kompensierender Aufbau noch fehlender demokratischer Strukturen auf der Ebene der Europäischen Union. Ob sich in dieser Lage die Völker Europas in der gegenwärtigen Situation schon auf dem langen Weg zur Verschmelzung zu einem Europäischen Volk befinden, um sich als solches demokratisch selbstbestimmen zu können, oder ob sie eher noch, um wahrhaft Demokratien bleiben zu können, eine Renationalisierung von Entscheidungsbefugnissen erstreben, ist einstweilen offen. Höchst unwahrscheinlich aber dürfte sein, daß sich die Völker Europas, solange sie sich als kategoriale politische Größe begreifen, einer Form der Fremdbestimmung unterwerfen werden. Ein von Technokraten herbeigezwungenes und beherrschtes Europa wird es als legitimes nicht geben. Wohlmeinende Rechtfertigungen solcher unübersehbaren Tendenzen könnten latentes Revolutionspotential schüren, das sich beim Scheitern einer kompetentiell entgrenzten und damit demokratisch nicht legitimierten europäischen (Währungs-) Politik unversehens entladen könnte. 